\title{
Towards a semiclassical understanding of chaotic single- and many-particle quantum dynamics at post-Heisenberg time scales
}

\author{
Daniel Waltner $\odot$ \\ Fakultät für Physik, Universität Duisburg-Essen, Lotharstraße 1, D-47048 Duisburg, Germany \\ Klaus Richter \\ Institut für Theoretische Physik, Universität Regensburg, D-93040 Regensburg, Germany
}

(Received 12 April 2019; published 17 October 2019)

\begin{abstract}
Despite considerable progress during the past decades in devising a semiclassical theory for classically chaotic quantum systems a quantitative semiclassical understanding of their dynamics at late times (beyond the so-called Heisenberg time $T_{H}$ ) is still missing. This challenge, corresponding to resolving spectral structures on energy scales below the mean level spacing, is intimately related to the quest for semiclassically restoring unitary quantum evolution. Guided through insights for quantum graphs we devise a periodic-orbit resummation procedure for spectra of quantum chaotic systems invoking periodic-orbit self-encounters as the structuring element of a hierarchical phase space dynamics. Quantum unitarity is reflected in real-valued spectral determinants with zeros giving discrete energy levels. We propose a way to purely semiclassically construct such real spectral determinants based on two major underlying mechanisms. (i) Complementary contributions to the spectral determinant from regrouped pseudo-orbits of duration $T<T_{H}$ and $T_{H}-T$ are complex conjugate to each other. (ii) Contributions from long periodic orbits involving multiple traversals along shorter orbits cancel out. We furthermore discuss implications for interacting $N$-particle quantum systems with a chaotic classical large- $N$ limit that have recently attracted particular interest in the context of many-body quantum chaos.
\end{abstract}

DOI: 10.1103/PhysRevE.100.042212

\section{INTRODUCTION}

The close connection between the energy level spectra of quantum systems with a chaotic classical limit and those resulting from random matrix theory was originally found by Bohigas, Giannoni, and Schmit [1], who conjectured that the level statistics of classically chaotic quantum systems obeys universal random matrix predictions. Experimental investigations of this level statistics have been performed for a wide range of systems ranging from typical many-body systems like nuclei [2] over single particles in chaotic billiards [3,4] to acoustic resonances [5]. One major approach to justify this conjecture is based on periodic orbit (PO) expansions of the spectral density (in terms of the Gutzwiller trace formula [6]) or related quantities such as the spectral determinant or zeta function [7-13]. For certain systems such expansions are exact, for example, for the dynamics on surfaces of constant negative curvature [14]. Generally, such PO expansions represent appropriate approximations to quantum spectra in the semiclassical limit that is defined by the condition that actions (of the shortest POs) in the corresponding classical system are much larger than the action quantum $\hbar$.

Furthermore, correlation functions of the spectral density or spectral determinant, both as functions of the energy $E$, can then be expressed in terms of multiple sums over contributions from the POs of the considered system. Each of the summands involved carry phases depending on the difference of the actions of different POs divided by $\hbar$. As long as the POs involved are classically uncorrelated this results in (random) phases rapidly varying as a function of $E$ such that the corresponding joined PO contributions average out. This leads to the quest for identifying pairs of correlated POs with nonrandom phase differences that survive the energy averages involved. This program was initiated by Berry [15] showing by means of the Hannay-Ozorio de Almeida sum rule [16] that the leading-order contribution to the spectral form factor, i.e., the Fourier transform of the spectral two-point autocorrelation function, is obtained by pairing identical trajectories, known as the so-called diagonal approximation. This approach is adequate for POs with periods $T$ considerably smaller than the Heisenberg time $T_{H}$. This corresponds to the time dual to the mean energy level spacing $\delta(E)$, i.e., the inverse of the mean level density $\bar{\rho}(E)$.

Further semiclassical contributions to the form factor for time scales $\tau=T / T_{H}<1$ result from pairs of POs linked to each other via close self-encounters where the periodic trajectories approach and depart from each other exponentially fast in the corresponding so-called encounter regions. The structure of the underlying trajectories was identified in Ref. [17] for the leading-order off-diagonal contribution to the spectral form factor. All off-diagonal higher-order corrections were eventually treated in [18] leading to the complete result for the spectral form factor for $\tau<1$, in accordance with random matrix theory. The latter work also established an analogy between PO diagrams and diagrams occurring in a perturbative expansion of the corresponding field theory. This concept found many applications in mesoscopic physics; see, e.g., Refs. [19-23] studying mainly the impact of a nonzero Ehrenfest time [24], a parameter not present in the random matrix theory description. 
The remaining high challenge has been to investigate the regime of times $T>T_{H}$, corresponding to semiclassically resolving spectral structures beyond the mean level spacing. If this were possible, this would, in principle, enable one to purely semiclassically compute individual energy levels, at least approximately. This was demonstrated in the past by using the cycle expansion [9], of which we will present here a refined version, for the helium atom [11] and the three-disk billiard $[10,13]$. Quantum mechanical unitarity and the discreteness of energy levels implies a saturation of the spectral form factor for $\tau>1$. Based on the semiclassical results for $\tau<1$ the form factor for $\tau>1$ was then formally obtained in a way that was inspired by a diagrammatic expansion of a four-point correlation function of spectral determinants around a second saddle point of the corresponding field theory [25]. Later on this procedure was connected to the use of the Riemann-Siegel lookalike formula for the spectral determinant [26] and to analytic continuation [27]. All such approaches have in common that they extend semiclassical results for $\tau<1$ to the regime $\tau>1$ by additionally invoking quantum mechanical unitarity in one or the other way. To the best of our knowledge a dynamical justification, i.e., a derivation entirely based on a semiclassical theory in terms of correlated POs, is however still lacking. This is much more than an academic problem and involves the deep and still open, far-reaching question in how far quantum unitarity can be achieved in an entirely semiclassical way, at least for chaotic quantum systems. This question is also particularly relevant for semiclassical calculations of the spectra of quantum chaotic systems themselves and not only for spectral correlations. Implications of unitarity were studied in this context in Refs. [28-32], and there exist also close connections to number theory $[12,33]$. Moreover, in the context of many-body and black hole physics the saturation and late-time behavior of the spectral form factor has recently attracted interest in theories with a gravitational dual motivated by the black hole information problem [34].

The developments mentioned above have lead to the belief that there is a complete analogy between field theory on the one side and semiclassical PO expansions on the other side. However, there are also important differences between the two methods: The effect of integrating over a curved manifold in field theory found its correspondence in the PO approach as contributions from long POs partially multiply following other shorter POs [35] (see, e.g., Fig. 1) referred to as partial repetitions in the following. Such configurations turn out to eventually cancel out for the form factor for $\tau<1[18,25]$. We will discuss them in detail below.

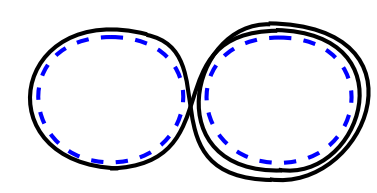

FIG. 1. Partial repetitions of a PO: A longer orbit (solid curve) comprising a 2-encounter formed by two shorter POs (dashed lines) traverses one of the shorter POs twice (dashed).
Quantum graphs [36] represent another class of systems where semiclassical approaches turn out to be exact and silent features of unitarity can be conveniently explored. Quantum graphs consist of a network of bonds along which the dynamics is usually determined by the one-dimensional Schrödinger equation. Different bonds are interconnected through vertices with couplings modeled by a unitary scattering matrix. Graphs have proven very useful as simple model systems in quantum chaos. For these systems a resummation procedure for the spectral determinant could be achieved that is purely based on the dynamics of the system [32]. As a key element it involves POs following shorter POs several times. It can thus be anticipated that contributions from different saddle points in field theory find their correspondence as orbits containing higher repetitions of shorter orbits in semiclassical PO expansions (as depicted in Fig. 1). For quantum graphs such configurations were shown to provide the underlying mechanism for the saturation of the form factor beyond $T_{H}$ [32].

However, as far as we know, such a correspondence has not yet been established for other chaotic systems, e.g., billiards, maps, and surfaces of constant negative curvature. Carrying over the procedure for graphs to the latter systems is not at all straightforward as the phases from the unitary scattering matrices at the vertices entering the spectral determinants of quantum graphs find no direct correspondence there.

The aim of this paper is to fill this gap: We suggest a corresponding resummation procedure for general chaotic systems. By this we provide the still missing correlations in the classical dynamics of chaotic systems responsible for the behavior of the two-point autocorrelation function in accordance with random matrix theory. By a Fourier transform this implies that the spectral form factor is given in the absence of time-reversal symmetry by

$$
K(\tau)=\tau \Theta(1-\tau)+\Theta(\tau-1) .
$$

In our derivation we require chaoticity (ergodicity and hyperbolicity) of the underlying classical dynamics such that a statistical description of close self-encounters is possible; for more details where the requirements become essential, see Ref. [18]. Furthermore, we assume of course the semiclassical limit $\hbar \rightarrow 0$ to hold. This implies a large mean level density $\bar{\rho}(E)$ and thus Heisenberg and Ehrenfest times much larger than typical classical time scales. In practice this limit $\hbar \rightarrow 0$ is realized by focusing on the part of the spectrum where $\bar{\rho}(E)$ is large. Within our approach we do not assume but provide a Riemann-Siegel lookalike relation for the spectral determinant indicating the validity of the BohigasGiannoni-Schmit conjecture [1] based purely on PO correlations without additionally invoking quantum mechanical unitarity.

The paper is organized as follows. In the next section we define the relevant quantities, explain what we mean by resummation, and recapitulate basic steps of the resummation procedure for quantum graphs. In Sec. III we show how to perform resummation for general chaotic systems. In Sec. IV we discuss implications for classically chaotic many-body quantum systems. We conclude in Sec. V followed by an Appendix containing technical details. 


\section{GENERAL ASPECTS OF RESUMMATION}

\section{A. Spectral determinant}

The spectral determinant is defined as (see, e.g., Ref. [12])

$$
\Delta(E)=\operatorname{det}[A(E, \hat{H})(E-\hat{H})]=\prod_{n=1}^{\infty} A\left(E, E_{n}\right)\left(E-E_{n}\right),
$$

with an appropriately chosen real regularizing function $A\left(E, E_{n}\right)$ that makes the product convergent. The zeros of the spectral determinant provide the energy eigenvalues $E_{n}$ of the corresponding quantum system which explains its central role. Various PO expansions of the spectral determinant have been considered (see, e.g., Refs. [7-10,12,13]). Within these approaches the spectral determinant is usually expressed as an infinite sum over classical pseudo-orbits $\Gamma$ (composite POs) of the underlying system [12]:

$$
\Delta(E)=e^{-i \pi \bar{N}(E)} \sum_{\Gamma}(-1)^{n_{\Gamma}} A_{\Gamma} e^{i S_{\Gamma}(E) / \hbar} .
$$

Here $\bar{N}(E)=\int_{-\infty}^{E} d E^{\prime} \bar{\rho}\left(E^{\prime}\right)$ is the average level counting function with $\bar{\rho}\left(E^{\prime}\right)$ the mean level density and $n_{\Gamma}$ denotes the number of composites of the pseudo-orbit $\Gamma$. Furthermore, $A_{\Gamma}$ is the product of the stability amplitudes of the orbits forming $\Gamma$ and $S_{\Gamma}(E)$ is the sum of the corresponding classical actions. We do not consider the contributions of real repetitions (multiple traversals of the entire POs) to Eq. (3) as they give subleading contributions [12]. Instead longer POs partially following shorter orbits several times as shown in Fig. 1 will prove relevant.

Expression (3) is not suitable for a numerical computation of the spectrum as it contains an infinite sum over POs. As their number increases exponentially with length, in analogy to the Riemann-Siegel resummation for the corresponding formula for the zeros of the Riemann zeta function [33], an expression containing a truncated sum was considered based on the fact that the expression (2) is real:

$$
\Delta_{\text {res }}(E)=e^{-i \pi \bar{N}(E)} \sum_{\Gamma, T \leqslant T_{H} / 2}(-1)^{n_{\Gamma}} A_{\Gamma} e^{i S_{\Gamma}(E) / \hbar}+\text { c.c. }
$$

The sum in Eq. (4) contains pseudo-orbits only up to half of the Heisenberg time that can be expressed in terms of the inverse mean level spacing as $T_{H}=2 \pi \hbar \bar{\rho}(E)$. Equation (4) was used successfully in the past to compute individual levels [37].

In the following we will refer to the procedure of transforming Eq. (3) into Eq. (4) as resummation. Without assuming that $\Delta(E)$ is real, that is without imposing unitarity, we will argue how to resum and add up the contributions from pseudo-orbits in a semiclassical framework in order to achieve $\Delta(E)$ to be real. Correspondingly, to explicitly show Eq. (4) consists of the following two essential steps that serve as a guideline for Sec. III.

(1) To demonstrate that the pseudo-orbit contributions to Eq. (3) with durations $T_{H} / 2<T_{\Gamma} \leqslant T_{H}$ provide a contribution to Eq. (3) complex conjugated to the one stemming from (complementary) pseudo-orbits with durations $0 \leqslant$ $T_{\Gamma}<T_{H} / 2$.
(2) To show that all contributions to Eq. (3) from pseudoorbits partially multiply traversing others mutually cancel.

In our computations to show the equivalence of Eqs. (3) and (4) we use the semiclassical limit $\hbar \rightarrow 0$ neglecting terms that are higher order in $\hbar$ and an average with respect to a quantum mechanically large but classically small energy window allowing one to neglect highly oscillating terms as a function of $E$.

\section{B. Resummation for quantum graphs}

As stated, Eq. (4) can be derived from Eq. (3) in a similar way as for the zeros of the Riemann zeta function (RiemannSiegel formula) using the fact that Eq. (2) is real. However, such a formal procedure does not provide any insight from a semiclassical perspective into the underlying PO structures, i.e., how the dynamics of the system and correlations between POs imply Eq. (4).

Such a connection was made for quantum graphs in $[32,38]$ extending earlier thorough studies for simple graphs in $[39,40]$ [as the one shown in Fig. 2(a)]. For quantum graphs the following resummation scheme arose: All contributions to the spectral determinant in Eq. (3) from pseudo-orbits involving multiple traversals of shorter orbits (similar to Fig. 1) cancel. This cancellation mechanism is related to the one found by Cvitanovic in the framework of the cycle expansion [9], but goes beyond that. This implies in particular for graphs, where the total bond length defines $T_{H}$, that POs with duration $T>T_{H}$ do not contribute, since they must necessarily contain partial repetitions. Moreover, contributions to the spectral determinant from pseudo-orbits without partial repetitions were found to be related to each other in a specific way: The contributions of pseudo-orbits covering a certain part of the graph are complex conjugated to the contributions from pseudo-orbits covering the complement (the part of the graph not covered by the pseudo-orbits mentioned first).

For an illustration of this mechanism consider the directed quantum graph in Fig. 2(b) containing the POs " 0 ", " 1 ", and " 2 " connected by the two vertices $a$ and $b$. In this case $A_{\Gamma}$ in Eqs. (3) and (4) is the product of the scattering matrix elements of the vertices traversed by $\Gamma$ [32]. We first address item (1) at the end of Sec. II A. To understand how complementary pseudo-orbit contributions add up we consider the specific unitary matrix

$$
\mathcal{C}=\frac{1}{\sqrt{2}}\left(\begin{array}{cc}
1 & 1 \\
-1 & 1
\end{array}\right)
$$

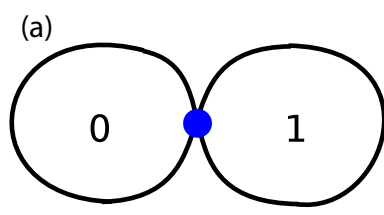

(b)

FIG. 2. Two simple quantum graph structures. In (a) a directed graph connects two bonds " 0 " and " 1 " at one vertex. In (b) the directed quantum graph consists of two vertices $a$ and $b$ connected by four bonds. 
defining the scattering at the vertex, as for this case we will find a direct correspondence between quantum graphs and general chaotic dynamics; see below.

The choice of $\mathcal{C}$ in Eq. (5) implies the following simple rule: Each time the (longer) orbit switches at a vertex between two (shorter) POs on the graph [for example, from "0" to "1" in the graph of Fig. 2(b) and back], its contribution to the spectral determinant involves the off-diagonal elements of $\mathcal{C}$ and acquires a minus sign, whereas a plus sign is acquired if there is no switching between POs. This implies that the contributions from orbit " 01 " and from the pseudoorbit consisting of " 0 " and " 1 " add up [the minus sign from $(-1)^{n_{\Gamma}}$ in Eq. (3) cancels with the one from the scattering matrix element]. The stability amplitudes of the sum of the contributions from " 01 " and " 0 ", " 1 " are thus equal to the amplitude of the complementary orbit " 2 ". The same holds true for periodic pseudo-orbits traversing four bonds. Here the contributions from the pseudo-orbits " 012 ", " 0 " " 12 ", " 2 " " 01 ", and " 0 " "1" "2" add up canceling the factor $\sqrt{2}^{-4}$ from the stability amplitudes. Taking into account that for the graph

$$
\pi \bar{N}(E)=\pi E \bar{\rho}(E)=\left(S_{0}+S_{1}+S_{2}\right) /(2 \hbar),
$$

the contributions to Eq. (3) from first " 01 " " 1 ", " 0 " and second "2" on the one hand and first " 012 ", " 01 " " 2 ", " 0 " " 12 ", "0" "1" "2" and second the pseudo-orbit containing no orbit on the other hand altogether add up to a real quantity.

A specific example for an orbit with multiple traversals is "001". Its contribution to Eq. (3) is canceled by the one from the pseudo-orbit consisting of " 0 " and of " 01 ", as both involve the traversals of the same vertices and bonds differing only by the factor $(-1)^{n_{\Gamma}}$. This illustrates item (2) pointed out at the end of the last subsection for this particular system.

\section{RESUMMATION FOR GENERAL CHAOTIC SYSTEMS}

\section{A. Outline}

We now generalize the procedure just outlined to chaotic dynamical systems. We first consider the statistics of 2encounters for a representative PO (not a pseudo-orbit) and will then introduce a resummation procedure along the lines of resummation outlined for quantum graphs.

The number $n$ of encounters for an orbit with $T=T_{H}$ can be estimated as follows. In general chaotic systems the stability amplitude $A_{\Gamma}$ introduced in Eq. (3) is given for large $T$ asymptotically by $e^{-\lambda T_{H} / 2}$. The number of pseudo-orbits without partial repetitions can be estimated by $2^{n}$-at every encounter of two POs as shown in Fig. 3 the longer orbit can decide to switch to another one or stay close to the first one. From the cancellation of these factors we obtain the condition

$$
e^{\lambda T_{H} / 2} \approx 2^{n}
$$

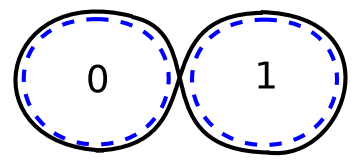

FIG. 3. Sketch of a PO possessing one 2-encounter as considered for resummation in Sec. III. for general chaotic systems, implying $n \approx \lambda T_{H} /(2 \ln 2)$ or

$$
n \approx d / \hbar
$$

with a classical constant $d$. Note that this estimate for $n$ is consistent with the one that follows from ergodicity arguments given in [17], if PO pairs with action differences up to order $\hbar$ are taken into account; for details see the Appendix.

In the following subsections we explain how to perform resummation, corresponding to the two steps at the end of Sec. II A, for general chaotic systems. Our reasoning is based on the statistical description of close encounters of POs developed in Refs. [18,25,35]. To make the presentation more transparent, we split it into three parts according to the following subsections.

In Sec. III B we ignore the relation (8) for the moment and restrict ourselves to the case that POs of duration $T_{H}$ possess at most one encounter. Hence diagrams with more than one encounter are not considered here and will be treated later. We demonstrate how in this case resummation can be performed in a similar way as in the case of a quantum graph containing a single vertex. More precisely, there we show how the contributions to the spectral determinant add up for durations equal to the Heisenberg time [contained in case (1) in Sec. II A] and how contributions from pseudo-orbits with partial repetitions cancel [case (2)].

In Sec. IIIC we then consider orbits with duration $T_{H}$ possessing at most two encounters. Here we again establish the analogy to resummation for graphs containing two vertices. We show how the contributions add up at durations $T=T_{H}$ and additionally in the range $T_{H} / 2<T<T_{H}$ [case (1)]. Case (2) can be shown in the same way as in Sec. III B.

In the last subsection we eventually work out the analogy between resummation for general chaotic systems and for quantum graphs for arbitrary $n$. This also includes $n$ values consistent with Eq. (8) using the results from the previous subsections.

\section{B. Orbits with at most one 2-encounter}

We first consider orbits (as the two dashed ones in Fig. 3) possessing at most one close encounter in phase space, i.e., $n \leqslant 1$, that can be constructed in hyperbolic systems. As established in the literature $[18,25]$ the distance between the orbit segments in phase space close to the encounter is measured along the directions of the stable and unstable manifold of one of the shorter orbits shown as dashed lines in Fig. 3. The distances are denoted by $s$ and $u$ for the stable and unstable direction, respectively.

As outlined at the end of Sec. II A, for the resummation we need to distinguish two scenarios that require different treatment. The first possibility contains contributions that add up from different pseudo-orbits, based on Fig. 3. The second one involves orbits with higher partial repetitions along at least one of the underlying short orbits indicated by dashed lines; an example is plotted in Fig. 1.

We start with the first scenario considering the pseudoorbit pair in Fig. 3. The long "eight-shaped" PO " 01 " is assumed to possess the period $T_{01}=T_{H}$ as we consider it as the reference filling the complete phase space without any partial repetitions. The location of its 2-encounter divides the 
orbit " 01 " into two pieces of durations $T_{0}$ and $T_{1}$ of roughly the same order with $T_{0}+T_{1} \approx T_{01}$. We further need to calculate the overall contribution to the spectral determinant (3) of these two partner trajectories. To this end we first summarize how the small difference between the pseudo-orbit, composed of " 0 " and " 1 ", and the long orbit is calculated on average in a statistical way. It is quantified in terms of the distances along the stable and unstable directions $s$ and $u$ in a Poincaré surface of section placed within the region where the orbits " 0 " and " 1 " encounter each other. Here the action difference $\Delta S=s u$ $[18,25]$ as well as the weight function $w_{01}(s, u)$ determining the probability for such an encounter to occur in a single PO is required. Using hyperbolicity the duration of the encounter between the two short orbits " 0 " and " 1 ",

$$
t_{\mathrm{enc}}=\frac{1}{\lambda} \ln \left(\frac{c^{2}}{|s u|}\right),
$$

is obtained. It corresponds to the Ehrenfest time in chaotic systems for $\Delta S \simeq \hbar$. In Eq. (9), $\lambda$ is the Lyapunov exponent and $c$ a classical constant that determines the upper limit for the coordinates $s$ and $u$ allowing for linearization of the dynamics along " 0 " around the one on " 1 ". The parts of the orbits " 0 " and " 1 " inside this linearizable region are commonly referred to as encounter stretches. In ergodic and hyperbolic systems the weight function is given by [18]

$$
w_{01}(s, u)=\frac{T_{01}\left(T_{01}-2 t_{\mathrm{enc}}\right)}{2 \Omega t_{\mathrm{enc}}},
$$

with $\Omega$ the volume of the surface of constant energy of the underlying system. This allows us to rewrite the common contribution from the orbit " 01 " and the pseudo-orbit consisting of " 0 " and " 1 " to the spectral determinant in Eq. (3) as

$$
A_{01} e^{i S_{01} / \hbar}-A_{0} A_{1} e^{i\left(S_{0}+S_{1}\right) / \hbar}=A_{0} A_{1} e^{i\left(S_{0}+S_{1}\right) / \hbar}\left(e^{i \Delta S / \hbar}-1\right),
$$

with $\Delta S=S_{01}-S_{0}-S_{1}$. Note that the orbits "0" and "1" are two representatives of the pseudo-orbits $\Gamma$ in Eq. (3) that we sum over when computing the spectral determinant. We consider the sum in Eq. (3) to act as an average over many such pairs (this will become more clear below). This allows for replacing in Eq. (11) the phase containing the action difference by its average using the weight function (10) and $\Delta S=s u$. Then we obtain

$$
\begin{aligned}
& A_{0} A_{1} e^{i\left(S_{0}+S_{1}\right) / \hbar}\left(\int_{-c}^{c} d s d u w_{T_{01}}(s, u) e^{i \Delta S / \hbar}-1\right) \\
& \approx-A_{0} A_{1} e^{i\left(S_{0}+S_{1}\right) / \hbar}\left(\frac{T_{01}}{T_{H}}+1\right) .
\end{aligned}
$$

In the last step we employed the relation

$$
\int_{-c}^{c} d s d u w_{01}(s, u) e^{i s u / \hbar}=-\frac{T_{01}}{T_{H}},
$$

obtained after averaging with respect to a classically small energy window (or equivalent duration window $\Delta T_{01}$ ) [18] leading to the fact that effectively only action differences of the order $\hbar$ contribute. Note that only the second term in the bracket in Eq. (10) contributes to Eq. (13) and the relation $T_{H}=\Omega /(2 \pi \hbar)$ was used.
It is instructive to compare these results with the results in Ref. [32] for the quantum graph in Fig. 2(a). Denoting as in Ref. [32] a contribution to the sum in Eq. (3) by

$$
t_{\Gamma}=(-1)^{n_{\Gamma}} A_{\Gamma} e^{i S_{\Gamma} / \hbar},
$$

we have the contributions from orbits in the graph that do not involve repetitions, i.e., the POs $t_{0}, t_{1}, t_{01}$ and the pseudo-orbit $t_{0} t_{1}$. In order to relate in this case Eq. (3) to Eq. (4) we need to consider $t_{01}$ and $t_{0} t_{1}$ using Eq. (5):

$t_{01}+t_{0} t_{1}=-\left(\frac{1}{2}+\frac{1}{2}\right) e^{i\left[S_{0}(E)+S_{1}(E)\right]}=-e^{i\left[S_{0}(E)+S_{1}(E)\right]}$,

with $T_{0}+T_{1}=T_{H}$. This contribution yields, together with the one from its complement, the pseudo-orbit of length zero, a real contribution to Eq. (3) when taking into account the corresponding relation (6) in this case.

Most notably, the same structure of signs is obtained in Eq. (12) arising from the minus sign of the encounter integral (13). The minus sign occurring from the encounter integral takes the same distinct role as the minus sign in the scattering matrix (5) in quantum graphs required for unitarity. Hence one can regard the minus sign arising from the encounter integral to be at the heart of a subtle semiclassical mechanism to achieve unitary behavior. To conclude this part, in a chaotic system the semiclassical contributions to the spectral determinant from the connected orbits add up with those from the disconnected ones in configurations shown in Fig. 3 yielding the common term (12).

In the next step we show that each orbit $p$ of duration $T_{p}$ longer than $T_{H}$ indeed has at least one encounter involving a multiple traversal of a shorter orbit. This follows from a closer inspection of the case where one of the short orbits is multiply traversed as in Fig. 1. The corresponding weight function $v_{T_{p}}(s, u)$ for that event can be obtained by similar arguments as in Ref. [18]; see also Ref. [35]: There for an ergodic system the number of piercing points through the Poincaré surface of section in the encounter region in the intervals $(s, s+d s)$ and $(u, u+d u)$ of stable and unstable coordinates in a time interval $(t, t+d t)$ is shown to be

$$
\frac{1}{\Omega} d s d u d t .
$$

The weight function $v_{T_{p}}(s, u)$ is then obtained by integrating this expression over all allowed values of $t$ and multiplying it by $T_{p} / t_{\text {enc }}$ in order to take into account all possible positions of the first encounter stretch. For $v_{T_{p}}(s, u)$ the variable $t$ in Eq. (16) has to be integrated from zero to $t_{\mathrm{enc}}$ as the encounter stretches are required to overlap, i.e., we claim that one of the two short orbits in Fig. 1 is multiply traversed by the long orbit with $T_{p}>T_{H}$ yielding

$$
v_{T_{p}}(s, u)=\frac{T_{p} t_{\mathrm{enc}}}{t_{\mathrm{enc}} \Omega} .
$$

This weight function allows us to evaluate the sum

$$
\sum_{i} e^{i s_{i} u_{i} / \hbar}
$$

running over all encounters of an orbit involving multiple traversals with action difference $\Delta S_{i}=s_{i} u_{i}$ up to order $\hbar$ $[20,41]$. Replacing this sum by an integral over $s$ and $u$, and 
employing Eq. (17), we get

$$
\int_{-c}^{c} d s d u v_{T_{p}}(s, u) e^{i s u / \hbar}=\int_{0}^{c} d s \frac{4 \hbar T_{p}}{\Omega s} \sin \frac{s c}{\hbar}=\frac{T_{p}}{T_{H}},
$$

where we used the semiclassical approximation $c^{2} / \hbar \gg 1$ in the last step. Note that, in contrast to Eq. (13), in Eq. (19) no average with respect to $\Delta T_{p}$ is needed for calculating the integral. The expression in Eq. (19) follows from Eq. (18) by an average using the distribution function $v_{T_{p}}(s, u)$. According to the right hand side of (19) the absolute value of expression (18) yields a number larger than one for $T_{p}>T_{H}$, implying that each orbit has on average at least one encounter involving a partial repetition. Then no contributions to Eqs. (3) and (4) arise for $T_{p}>T_{H}$ as we will show in the following.

Turning to case (2) defined at the end of Sec. II A we are concerned with the situation where at least one of the orbits " 0 " and " 1 " is multiply traversed. Then in the case of graphs the connection between Eq. (3) and Eq. (4) results from the cancellation of the contribution from a given orbit (with multiple partial traversals) by the contribution from a corresponding pseudo-orbit where one of the multiply traversed suborbits is split off [32]. For instance, the orbit "001" is canceled by the pseudo-orbit consisting of the orbits " 0 " and "01". In such cases both components, here "001" as well as " 0 " and " 01 ", exhibit the same number of transitions between " 0 " and " 1 ".

Notably, the same cancellation holds true for general chaotic dynamics. To show this we split the long POs in Figs. 1 and 3 into their components " 0 " and " 1 " getting

$$
\begin{aligned}
& A_{001} e^{i S_{001} / \hbar}-A_{01} A_{0} e^{i\left(S_{01}+S_{0}\right) / \hbar} \\
& \quad \approx A_{0}^{2} A_{1} e^{i\left(2 S_{0}+S_{1}\right) / \hbar}\left(e^{i \Delta S_{1} / \hbar}-e^{i \Delta S_{2} / \hbar}\right) .
\end{aligned}
$$

Here $\Delta S_{1}$ is the action difference between the PO " 001 " and the pseudo-orbit consisting of " 0 ", " 0 ", and " 1 " and, correspondingly, $\Delta S_{2}$ is the action difference between the pseudo-orbits " 01 " and " 0 " and again " 0 ", " 0 ", and " 1 ". The action differences are given by $[20,41]$

$$
\Delta S_{1}=s u+s u e^{-\lambda T_{0}}, \quad \Delta S_{2}=s u .
$$

The terms $s u$ in $\Delta S_{1}$ and $\Delta S_{2}$ are determined only by the difference in the number of respective transitions between " 0 " and " 1 " of the relevant pseudo-orbits and are thus the same for $\Delta S_{1}$ and $\Delta S_{2}$. Only the second term in $\Delta S_{1}$ depends on the details of how the corresponding pseudo-orbit surrounds "0" and "1". Following the same reasoning as above Eq. (12) we again replace the latter expression by its average using the weight function (10) and obtain

$$
\begin{aligned}
& A_{0}^{2} A_{1} e^{i\left(2 S_{0}+S_{1}\right) / \hbar} \int_{-c}^{c} d s d u w_{T_{p}}(s, u)\left(e^{i \Delta S_{1} / \hbar}-e^{i \Delta S_{2} / \hbar}\right) \\
& =O\left(e^{-\lambda T_{0}}\right) .
\end{aligned}
$$

The right hand side (RHS) follows from Taylor expanding the exponential containing the second term in $\Delta S_{1}$ and is negligible for $T_{0} \sim T_{1} \sim T_{H}$. This implies a cancellation of the corresponding contributions to the spectral determinant, that is, all contributions from POs with one 2-encounter and multiple partial traversals along one of the shorter POs involved.
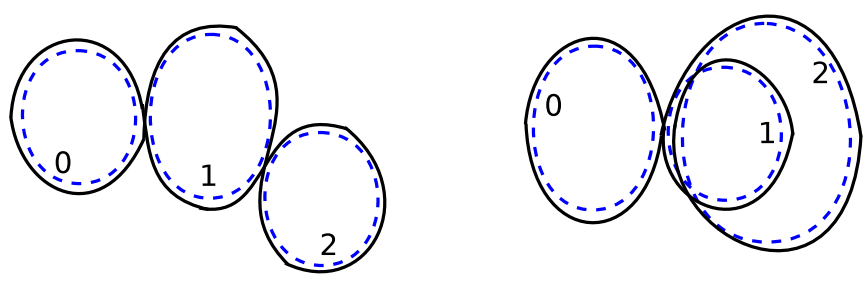

FIG. 4. Long PO (solid curve) composed of three shorter POs " 0 ", "1", and "2" (dashed) by means of two separate (left) or overlapping (right) 2-encounters.

\section{Orbits with at most two 2-encounters $(n \leqslant 2)$}

Here we generalize the statements from the last subsection to POs with at most two encounters. We include this intermediate step before addressing the general case of at most $n$ encounters [with $n$ integer usually of the order $1 / \hbar$ for $T \sim$ $T_{H}$; see Eq. (8)], because it is still much more transparent than the general case, and it provides additional insights compared to the above case $n \leqslant 1$.

We first note that we do not require the two 2-encounters to be separated from each other but also allow them to overlap, see right panel of Fig. 4, in contrast to the nomenclature from previous literature $[18,25]$ where this situation is referred to as a 3-encounter. Again we first consider item (1) at the end of Sec. II A. Using the same arguments as above given between Eqs. (11) and (12) we obtain for the contribution (of PO configurations as in Fig. 4) to the spectral determinant

$$
\begin{aligned}
& A_{012} e^{i S_{012}}-A_{01} A_{2} e^{i\left(S_{01}+S_{2}\right) / \hbar} \\
& \quad-A_{12} A_{0} e^{i\left(S_{12}+S_{0}\right) / \hbar}+A_{0} A_{1} A_{2} e^{i\left(S_{0}+S_{1}+S_{2}\right) / \hbar} \\
& \quad \approx A_{0} A_{1} A_{2} e^{i\left(S_{0}+S_{1}+S_{2}\right) / \hbar}\left[1+2 \frac{T_{012}}{T_{H}}+\left(\frac{T_{012}}{T_{H}}\right)^{2}\right] .
\end{aligned}
$$

In the equation above we get two contributions from pseudoorbits consisting of two orbits. Note that in the case of large $n$ discussed below we obtain a sum over the contributions from many different encounters for the same constituents. The fact that $n$ is eventually large justifies the averaging by $w_{01}(s, u)$ introduced above. Expression (22) also holds true for overlapping encounters (Fig. 4, right), as only the (still) connected and the split off orbit need to be distinguished. Comparing the results above to the ones for the pseudo-orbits formed by " 0 ", " 1 ", " 2 " on quantum graphs we again find direct correspondence to the ones in Eq. (22) for $T_{012}=T_{H}$; concerning the stability amplitudes remember Eq. (7).

Contrary to the last subsection (where only " 0 " and " 1 " were occurring with durations between $T_{H} / 2$ and $T_{H}$ hence not allowing for resummation) here several pseudo-orbits exist with such durations consisting of three bonds for the corresponding graphs. As shown in Sec. II B the two contributions (from one fully connected PO and from a pseudo-orbit with two components) can be directly added up rendering these contributions equal to the complementary ones from orbits consisting of one bond.

Turning to general chaotic systems we obtain by a calculation analogous to the one in Eq. (22) powers of ratios of $T_{012} / T_{H}$ in that case. In chaotic systems where the encounters are distributed in an ergodic manner such a ratio can be 
interpreted as the probability for an orbit "012" (denoted here by $p$ ) of duration $T_{p}$ to reach a certain encounter (instead of $T_{H}$ where the encounter is reached for sure). We relate this contribution from pseudo-orbits of duration $T_{p}$ to the one from complementary pseudo-orbits of duration $T_{p}^{\prime}=T_{H}-T_{p}$. Their stability amplitudes are $2^{-\left(T_{H}-T_{p}\right) /(2 \bar{T})}$ and $2^{-T_{p} /(2 \bar{T})}$, respectively, with $\bar{T}$ the average temporal distance between encounters. The additional number of the pseudo-orbits that can be formed in the latter case is given by $2^{\left(T_{p}-T_{H} / 2\right) / \bar{T}}$. This number is obtained as follows. The duration difference between $\left(T_{H}-T_{p}\right) / 2$ and $T_{p} / 2$ is $T_{p}-T_{H} / 2$. The number of encounters traversed during this time is $\left(T_{p}-T_{H} / 2\right) / \bar{T}$. At each encounter the trajectory branches are associated with two possibilities, in total $2^{\left(T_{p}-T_{H} / 2\right) / \bar{T}}$ possibilities. Multiplying this number by the stability amplitude $2^{-T_{p} /(2 \bar{T})}$ of an orbit with duration $T_{p}$ we obtain the amplitude of an orbit of duration $\left(T_{H}-T_{p}\right) / 2$. Taking into account that the actions of the pseudo-orbits of durations $T_{p}$ and $T_{p}^{\prime}$ sum up to the action of an orbit of duration $T_{H}$ we see in view of relation (6) that these two contributions to the spectral determinant are indeed complex conjugate to each other.

Orbits involving multiple traversals of shorter orbits again do not contribute to the spectral determinant due to the same arguments as given in the preceding subsection.

\section{Orbits with at most $\boldsymbol{n}$ 2-encounters}

Here we consider resummation for the general case of a system with at most $n$ 2-encounters. We analyze exemplarily the case of one fully connected orbit without repetitions; see the left diagram in Fig. 5. We will comment on how to generalize this analysis also to cases where several connected orbits exist, e.g., the right diagram in Fig. 5, at the end of this subsection.

For the case of a fully connected PO with at most $n$ two 2-encounters we can proceed in an analogous manner as above. At first, we have one fully connected orbit obtained by combining the pseudo-orbits at all encounters. Furthermore, consider pseudo-orbits composed of two components where at all encounters except for one the (shorter) pseudo-orbits are combined; we have $n$ possibilities to choose the encounters where the pseudo-orbit is already combined. This factor $n$ replaces the factor 2 obtained in Eq. (22) in Sec. III C. Considering pseudo-orbits consisting of three components we have $n(n-1) / 2$ possibilities to choose the encounters where the
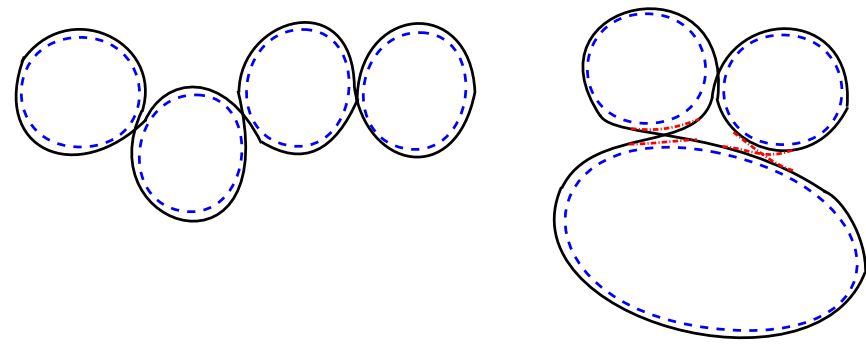

FIG. 5. Left panel: One long, fully connected PO (full line) surrounds all shorter POs (dashed lines). Right panel: Two different configurations of connected orbits can be constructed surrounding the shorter POs (indicated by the full and dashed dotted lines). orbit is not split to obtain the pseudo-orbit: $n$ for choosing the first encounter with combination of pseudo-orbits and $n-1$ for choosing the second. The factor $1 / 2$ enters because they cannot be distinguished. This procedure can be carried on for pseudo-orbits consisting of more components. As shown for graphs in Ref. [32] we thus get a coherent sum of contributions from pseudo-orbits containing no partial repetitions. Their overall number is

$$
\sum_{v=0}^{n}\left(\begin{array}{l}
n \\
v
\end{array}\right)=2^{n}
$$

where the contributions explained in Eq. (22) correspond to $n=2$ in the equation above. Using Eq. (7), the overall factor $2^{n}$ in Eq. (23) again cancels the stability amplitude $2^{-n}$ resulting from the $2 n$ links between the encounters in this case. This leads again to two complementary contributions to the spectral determinant complex conjugated to each other yielding together a real result.

Here we assumed that the exponential increase in the number of POs of duration $T$ proportional to $e^{\lambda T}$ is caused by branching of the POs at the $n$ encounters (with action differences of order $\hbar$ ). Such an assumption [see also Eq. (7)] is safely fulfilled for long orbits of durations of order $T_{H}$ as they cover the energy shell on scales of order $\hbar$ leading to encounters with action differences that small that they contribute to Eqs. (13) and (19).

As mentioned at the beginning of this subsection this analysis was done for graphs with only one fully connected orbit traversing the full graph without repetitions. As we know first from our calculations for graphs [32] that the resummation can be done for several fully connected orbits without repetitions covering the full graph in a similar way, provided we obtain a minus sign when splitting the orbit at a 2 -encounter, and second that we have in the case of continuous dynamics the same ingredient, i.e., a minus sign resulting from splitting the orbit at every 2 -encounter, the resummation can be performed in the same way for continuous dynamics as for graphs.

Concerning the resummation of pseudo-orbits shorter than the $T_{H}$ we can proceed in the very same way as described in the last subsection. The same also applies to the cancellation of the contribution from POs with partial repetitions.

Throughout the above derivations we showed the analogy between the resummation procedures for quantum graphs on the one side and for systems with chaotic dynamics on the other side. These arguments hold for arbitrary values of $n$ and thus also for values consistent with Eq. (8).

\section{IMPLICATIONS FOR QUANTUM CHAOTIC MANY-BODY DYNAMICS}

So far we implicitly assumed chaotic dynamics in the lowdimensional phase space of single-particle systems. However, the semiclassical methods forming the basis of our present work are not limited to single-particle dynamics: Recently, interacting many-body (MB) quantum systems with a chaotic classical limit have gained considerable interest across different communities in theoretical physics. In the MB context a semiclassical version of the Gutzwiller-van Vleck propagator was derived for bosonic [42] and fermionic [43] MB systems, 
as well as for spin systems [44]. Specifically, periodic-orbit expansions could be deduced for the density of states of interacting bosonic quantum fields on a lattice $[45,46]$. These are applicable under the following assumptions. First, the semiclassical regime corresponds to the thermodynamic limit of large particle numbers $N \gg 1$, where $1 / N$ takes the role of an effective $\hbar$. In the classical (mean-field) limit, reached for $N \rightarrow \infty$, these systems are described by nonlinear wave equations. Second, we assume this mean-field dynamics to be fully chaotic, i.e., ergodic and hyperbolic. The prerequisites of the latter assumption are the subject of current research. For example, in Ref. [47] the hyperbolicity of mean-field dynamics corresponding to the Bose-Hubbard Hamiltonian was studied. With these assumptions, it was shown in Refs. [45,46] that the fluctuating part of the bosonic MB density of states can then be represented in terms of a semiclassical trace formula that has the very same structure as Gutzwiller's trace formula [6] for chaotic single-particle quantum systems. Periodic meanfield solutions of the nonlinear wave equations take the role of the classical single-particle POs in Gutzwiller's trace formula, including corresponding stabilities and actions. This close formal analogy has then allowed for rather straightforwardly generalizing the semiclassical calculation of the spectral MB form factor $[45,46]$, more generally, providing a dynamical explanation of universal spectral statistics in chaotic MB systems [48]. To this end a real-valued MB spectral determinant was again assumed and employed [46].

Due to this formal analogy between the semiclassical treatment of single-particle and MB dynamics, our arguments and derivations, put forward in Sec. III, providing a semiclassical interpretation of the real valuedness of the spectral determinant can be taken over to the MB case. The main underlying aspect involves a hierarchical structure of POs, intertwined through encounters that provide the mechanism for long POs to be composed of (multiple repetitions along) shorter POs. In the MB context corresponding encounters exist between (periodic) mean-field modes in the high-dimensional MB phase space and provide the underlying semiclassical mechanism for true quantum MB interference. In other words, in large- $N$ quantum systems entanglement is created (at time scales beyond the Ehrenfest time $\log N$ ) between different mean-field solutions as they are linked to each other through encounters [51]. It has been recently shown [52] that corresponding semiclassical techniques indeed quantitatively describe MB quantum interference at such post-Ehrenfest time scales.

Under the assumption of chaotic mean-field dynamics and $N \gg 1$, this altogether indicates to reinterpret items (1) and (2) in Sec. II A as follows.

(1) Contributions to the MB spectral determinant from pseudo-orbits (based on periodic mean-field solutions) of duration $T<T_{H}$ are complex conjugate to contributions from pseudo-orbits of length $T_{H}-T$ covering the complementary MB phase space area.

(2) All contributions to the MB spectral determinant from pseudo-orbits involving multiple partial traversals mutually cancel. In particular, this implies that pseudo-orbits with durations $T>T_{H}$ that necessarily involve such multiple traversals do not contribute.

These items allow one to show that spectral correlations are consistent with random matrix theory in a similar way as in
Ref. [46]. This is compatible with the results from numerical investigations for various many-body systems [46,53]. The latter results can be seen as another independent justification for our assumption of chaotic mean-field dynamics.

\section{CONCLUSIONS}

In this paper we have demonstrated how to generalize a resummation procedure for pseudo-orbits in quantum graphs to general quantum mechanical single-particle systems with chaotic classical counterparts, with further implications to chaotic MB systems. In the semiclassical limit long orbits with durations of the order of or larger than the Heisenberg time fill the phase space so densely that the previously considered individual encounters lose their significance and are replaced by POs containing partial repetitions from surrounding shorter POs. Thereby we demonstrate a corresponding Riemann-Siegel relation for the spectral determinant exploiting subtle classical correlation mechanisms of periodic pseudo-orbits. Using this relation and following the calculation in Ref. [26] we can further justify the validity of the Bohigas-Giannoni-Schmit conjecture based on purely dynamical grounds. Establishing a dynamical mechanism for a real-valued spectral determinant moreover implies a better understanding of the spectral form factor and its saturation in the late time limit beyond the Heisenberg time, based on purely semiclassical arguments, also with possible implications for black hole physics [34].

The analogy to quantum graphs shown in this paper was established for chaotic systems without time-reversal symmetry and graphs with directed bonds. However, it can be generalized in a straightforward manner to systems with timereversal symmetry. In that case, apart from the pseudo-orbit pairs existing without time-reversal symmetry, there exist additional orbit pairs traversed in opposite directions. Their contributions add up and cancel in the same way as explained in the main part of the paper. Additionally, two further possibilities arise, as the short multiply traversed orbits, e.g., "0" and " 1 " in Fig. 3, can traverse the encounter region relative to each other in two different directions. However, for these configurations we can identify further encounters in the same orbit where the direction of traversal relative to each other is not switched and then add up or cancel their contributions as described in the main part. Altogether the resummation procedure in the presence of time-reversal symmetry can be obtained as a straightforward extension of the one for systems without time-reversal symmetry. The same applies also to systems with weak spin-orbit coupling (see Ref. [54] for a semiclassical treatment) as in that case the classical dynamics remains unchanged.

It is instructive to compare the spectral determinant $(3,4)$ with the corresponding equivalents for the zeros of the Riemann zeta function. For the latter an equivalent to Eq. (4) with a variable cutoff and a corresponding error term resulting from the cutoff were derived [12]. This term is minimized by choosing $T_{H} / 2$ as cutoff. A corresponding error term for dynamical systems is not known. We think that a rigorous estimation of the error to obtain Eq. (4) from Eq. (3) should be possible within our approach as the errors connected with every step we perform are explicitly known. We thus 
consider our work as an appropriate basis for such further investigations.

Another aspect of this analogy between the spectral determinant for the Riemann zeta function and the one for dynamical systems arises in the context of the Hardy-Littlewood conjecture [55] describing off-diagonal correlations between prime numbers, the analog to periodic orbits (not pseudoorbits) for dynamical systems. Besides the periodic pseudoorbit expansions studied in the present article there exist also the expansions of the spectral form factor in terms of periodic orbits; see, e.g., Refs. $[15,17,18]$. By relating the pseudo-orbit contributions to each other, below and above $T_{H} / 2$, here we identified the corresponding off-diagonal pseudo-orbit correlations responsible for a behavior of the spectral form factor beyond $T_{H}$ consistent with random matrix theory. It would be very interesting to analyze the correlations between periodic orbits induced by these pseudo-orbit correlations, thereby eventually providing an analog to the Hardy-Littlewood conjecture for dynamical systems.

The Riemann-Siegel relation reflects unitarity and holds true independent of the character of the dynamics of the underlying classical system. However, the construction of the diagrams considered here assumes exponentially approaching and deviating trajectories as long as the dynamics is linearizable and ergodicity holds for the underlying system. It would be thus interesting to identify the relevant correlations in the case of integrable and mixed dynamics.

Moreover, as outlined in Sec. IV semiclassical techniques as the one used provide powerful tools to treat quantum MB systems with complex classical many-particle dynamics in the wide terrain of large particle number or small $\hbar$. Hence it is of interest to further make use of the benefits resulting from semiclassical resummation of the spectral determinant for the calculation of quantum spectra of many-particle systems with focus on effects typical for them such as indistinguishability of the particles or collective MB dynamics [56]. More generally, to better understand spectral features of the large variety of $\mathrm{MB}$ dynamics remains as a future challenge.

\section{ACKNOWLEDGMENTS}

One of us (K.R.) is very thankful to the late Bruno Eckhardt for many illuminating conversations about semiclassics over the years. We further thank Petr Braun and Gregor Tanner for numerous discussions and furthermore Petr Braun for many valuable suggestions to improve the presentation of this manuscript. We further acknowledge support by the Deutsche Forschungsgemeinschaft through Projects No. Gu431/9-1 and No. Ri681/14-1 (within the "Dreiburg cooperation").

\section{APPENDIX: ESTIMATE FOR THE NUMBER OF ENCOUNTERS}

We give an estimate for the number $n$ of encounters [see Eq. (8)] based on arguments given in Ref. [17]. There the number of self-crossings $d n$ of an orbit of duration $T$ under an angle in the range $[\epsilon, \epsilon+d \epsilon]$ was estimated for a billiard of area $A$ and $\epsilon \ll 1$ as

$$
d n \sim \frac{T^{2} v^{2}}{4 \pi A} d \epsilon^{2},
$$

with the magnitude of the velocity $v$. Employing the expression for the action difference

$$
\Delta S=\frac{p^{2} \epsilon^{2}}{2 m \lambda}
$$

with mass $m$ and Lyapunov exponent $\lambda$ between orbit and partner orbit in Fig. 3 the expression (A1) can be rewritten as

$$
d n \sim \frac{\lambda T^{2}}{2 \pi m A} d(\Delta S) .
$$

Taking into account only action differences up to order $\hbar$ we obtain by integration for the number of encounters of a PO at $T=T_{H}$

$$
n \sim \frac{\lambda T_{H}^{2} \hbar}{2 \pi m A}=\frac{\lambda T_{H}}{2 \pi}=\frac{m \lambda A}{2 \pi \hbar},
$$

providing Eq. (8) of the main text.
[1] O. Bohigas, M. J. Giannoni, and C. Schmit, Phys. Rev. Lett. 52, 1 (1984).

[2] R. U. Haq, A. Pandey, and O. Bohigas, Phys. Rev. Lett. 48, 1086 (1982).

[3] B. Dietz, A. Heine, A. Richter, O. Bohigas, and P. Leboeuf, Phys. Rev. E 73, 035201(R) (2006).

[4] H.-J. Stöckmann and J. Stein, Phys. Rev. Lett. 64, 2215 (1990).

[5] C. Ellegaard, T. Guhr, K. Lindemann, J. Nygård, and M. Oxborrow, Phys. Rev. Lett. 77, 4918 (1996).

[6] M. C. Gutzwiller, Chaos in Classical and Quantum Mechanics (Springer, Berlin, 1990).

[7] M. V. Berry and J. P. Keating, J. Phys. A 23, 4839 (1990).

[8] E. B. Bogomolny, Nonlinearity 5, 805 (1992).

[9] P. Cvitanović, Phys. Rev. Lett. 61, 2729 (1988).

[10] P. Cvitanović and B. Eckhardt, Phys. Rev. Lett. 63, 823 (1989).

[11] G. Ezra, K. Richter, G. Tanner, and D. Wintgen, J. Phys. B 24, L413 (1991).
[12] J. P. Keating, in Proceedings of the International School of Physics "Enrico Fermi," edited by G. Casati, I. Guarneri, and U. Smilansky (North-Holland, Amsterdam, 1993).

[13] A. Wirzba, Phys. Rep. 309, 1 (1999).

[14] N. L. Balazs and A. Voros, Phys. Rep. 143, 109 (1986).

[15] M. V. Berry, Proc. R. Soc. A 400, 229 (1985).

[16] J. H. Hannay and A. M. Ozorio de Almeida, J. Phys. A 17, 3429 (1984).

[17] M. Sieber and K. Richter, Phys. Scr. T 90, 128 (2001).

[18] S. Müller, S. Heusler, P. Braun, F. Haake, and A. Altland, Phys. Rev. Lett. 93, 014103 (2004); Phys. Rev. E 72, 046207 (2005).

[19] J. Kuipers, D. Waltner, C. Petitjean, G. Berkolaiko, and K. Richter, Phys. Rev. Lett. 104, 027001 (2010).

[20] P. W. Brouwer and S. Rahav, Phys. Rev. B 74, 075322 (2006).

[21] S. Müller, S. Heusler, P. Braun, and F. Haake, New J. Phys. 9, 12 (2007).

[22] R. S. Whitney and Ph. Jacquod, Phys. Rev. Lett. 96, 206804 (2006). 
[23] M. Novaes, Europhys. Lett. 98, 20006 (2012).

[24] B. V. Chirikov, F. M. Izrailev, and D. L. Shepelyansky, Sov. Sci. Rev., Sect. C 2, 209 (1981).

[25] S. Heusler, S. Müller, A. Altland, P. Braun, and F. Haake, Phys. Rev. Lett. 98, 044103 (2007).

[26] J. P. Keating and S. Müller, Proc. R. Soc. A 463, 3241 (2007).

[27] P. Braun and D. Waltner, J. Phys. A 52, 065101 (2019).

[28] M. Marden, Geometry of Polynomials (American Mathematical Society, Providence, RI, 1966).

[29] E. Bogomolny, O. Bohigas, and P. Leboeuf, Phys. Rev. Lett. 68, 2726 (1992); J. Stat. Phys. 85, 639 (1996).

[30] M. Kuś, F. Haake, and B. Eckhardt, Z. Phys. B 92, 221 (1993).

[31] F. Haake, M. Kuś, H.-J. Sommers, H. Schomerus, and K. Życkowski, J. Phys. A 29, 3641 (1996).

[32] D. Waltner, S. Gnutzmann, G. Tanner, and K. Richter, Phys. Rev. E 87, 052919 (2013).

[33] M. V. Berry and J. Keating, SIAM Rev. 41, 236 (1999).

[34] Ph. Saad, S. H. Shenker, and D. Stanford, arXiv:1806.06840.

[35] D. Waltner, S. Heusler, J. D. Urbina, and K. Richter, J. Phys. A 42, 292001 (2009).

[36] T. Kottos and U. Smilansky, Ann. Phys. (N.Y.) 274, 76 (1999); S. Gnutzmann and U. Smilansky, Adv. Phys. 55, 527 (2006).

[37] M. Sieber and F. Steiner, Phys. Rev. Lett. 67, 1941 (1991).

[38] R. Band, J. M. Harrison, and C. H. Joyner, J. Phys. A 45, 325204 (2012).

[39] H. Schanz and U. Smilansky, Philos. Mag. B 80, 1999 (2000).

[40] G. Tanner, J. Phys. A 33, 3567 (2000).

[41] J. Kuipers and M. Sieber, Phys. Rev. E 77, 046219 (2008).
[42] T. Engl, J. Dujardin, A. Argüelles, P. Schlagheck, K. Richter, and J. D. Urbina, Phys. Rev. Lett. 112, 140403 (2014).

[43] T. Engl, P. Plöß1, J. D. Urbina, and K. Richter, Theor. Chem. Acc. 133, 1563 (2014).

[44] D. Waltner, P. Braun, M. Akila, and T. Guhr, J. Phys. A 50, 085304 (2017).

[45] T. Engl, J. D. Urbina, and K. Richter, Phys. Rev. E 92, 062907 (2015).

[46] R. Dubertrand and S. Müller, New J. Phys. 18, 033009 (2016).

[47] S. Tomsovic, Phys. Rev. E 98, 023301 (2018).

[48] The conclusions drawn in Refs. [45, 46] coincide with recent results showing random-matrix behavior of the spectral form factor for a periodically kicked Ising spin model (to leading orders in $T / T_{H}$ ) [49] and a Floquet spin model [50].

[49] P. Kos, M. Ljubotina, and T. Prosen, Phys. Rev. X 8, 021062 (2018).

[50] A. Chan, A. De Luca, and J. T. Chalker, Phys. Rev. Lett. 121, 060601 (2018)

[51] J. Rammensee, J. D. Urbina, and K. Richter, Phys. Rev. Lett. 121, 124101 (2018).

[52] S. Tomsovic, P. Schlagheck, D. Ullmo, J. D. Urbina, and K. Richter, Phys. Rev. A 97, 061606(R) (2018).

[53] C. Pineda and T. Prosen, Phys. Rev. E 76, 061127 (2007).

[54] J. Bolte and S. Keppeler, Phys. Rev. Lett. 81, 1987 (1998); Ann. Phys. (N.Y.) 274, 125 (1999).

[55] G. H. Hardy and J. E. Littlewood, Acta Math. 44, 1 (1923).

[56] M. Akila, D. Waltner, B. Gutkin, P. Braun, and T. Guhr, Phys. Rev. Lett. 118, 164101 (2017). 